\title{
Frequently rearranged in advanced T-cell lymphomas-1 demonstrates oncogenic properties in prostate cancer
}

\author{
WEI ZHANG ${ }^{1}$, HUA XIONG $^{2}$, YANMEI ZOU ${ }^{2}$, SANPENG XU $^{3}$, LANPING QUAN ${ }^{4}$, \\ XIANGLIN YUAN ${ }^{2}$, NINGZHI XU ${ }^{4}$ and YIHUA WANG ${ }^{2,5}$ \\ ${ }^{1}$ Department of Urology, The Central Hospital of Wuhan, Wuhan, Hubei 430014; ${ }^{2}$ Department of Oncology, Tongji Hospital,
Tongji Medical College; ${ }^{3}$ Department of Pathology, Tongji Hospital, Huazhong University of Science and Technology,
Wuhan, Hubei 430030; ${ }^{4}$ Laboratory of Cell and Molecular Biology \& State Key Laboratory of Molecular Oncology,
Cancer Hospital, Chinese Academy of Medical Sciences \& Peking Union Medical College, Beijing 100021, P.R. China;
${ }^{5}$ Biological Sciences, Faculty of Natural and Environmental Sciences, University of Southampton, Southampton SO17 1BJ, UK
}

Received April 26, 2015; Accepted July 6, 2016

DOI: $10.3892 / \mathrm{mmr} .2016 .5704$

\begin{abstract}
Prostate cancer is the fifth most common cause of cancer-associated mortality for males worldwide. Although dysregulation of the $\beta$-catenin/T-cell factor (TCF) pathway has been previously reported in prostate cancer, the mechanisms underlying this process remain unknown. Frequently rearranged in advanced T-cell lymphomas-1 (FRAT1) functions as a positive regulator of the $\beta$-catenin/TCF signaling pathway. However, to the best of our knowledge, the molecular association between FRAT1 and the $\beta$-catenin/TCF pathway in prostate cancer has not been investigated. In the present study, FRAT1 expression was analyzed in normal prostate tissues and prostate adenocarcinoma samples using publicly available databases, a commercial tissue microarray and immunohistochemistry techniques. In addition, FRAT1 expression levels were altered by overexpression or RNA interference-mediated depletion in prostate cancer cells. The effects of FRAT1 expression on tumor growth were determined using cell growth curves in vitro and xenografts in nude mice in vivo. The effects of FRAT1 on $\beta$-catenin/TCF activity were measured using the TOPFLASH reporter assay. FRAT1 was expressed exclusively in the nuclei of normal prostate basal cells, and
\end{abstract}

Correspondence to: Dr Yihua Wang, Biological Sciences, Faculty of Natural and Environmental Sciences, University of Southampton, Life Sciences Building 85, Highfield Campus, Southampton SO17 1BJ, UK

E-mail: yihua.wang@soton.ac.uk

Professor Ningzhi Xu, Laboratory of Cell and Molecular Biology \& State Key Laboratory of Molecular Oncology, Cancer Hospital, Chinese Academy of Medical Sciences \& Peking Union Medical College, 17 Panjiayuan Nanli, Chaoyang, Beijing 100021, P.R. China

E-mail: xuningzhi@cicams.ac.cn

Key words: frequently rearranged in advanced T-cell lymphomas-1, prostate cancer, Wnt, $\beta$-catenin nuclear FRAT1 was detected in 68\% (40/59) of prostate adenocarcinoma samples. In addition, FRAT1 activated the TCF luciferase reporter gene promoter in prostate cancer cells, and was observed to promote the growth of prostate cancer cells in vitro. Furthermore, FRAT1 expression was sufficient to transform NIH3T3 mouse embryonic fibroblast cells and lead to tumor formation in vivo. These results suggest that FRAT1 demonstrates oncogenic properties in prostate cancer, potentially by suppressing the inhibitory effect of nuclear glycogen synthase $3 \beta$ against $\beta$-catenin/TCF activity, thus activating the Wnt/ $\beta$-catenin signaling pathway and promoting cell growth.

\section{Introduction}

Prostate cancer is the second most frequently diagnosed cancer in males worldwide, and is the most frequently diagnosed cancer among males in more developed countries (1). Despite improvements in the clinical management and treatment of prostate cancer, the five-year relative survival rate for patients with advanced prostate cancer is $\sim 30 \%$. It is estimated that 220,800 men in the USA developed prostate cancer during 2015 , and 27,540 men $(12.5 \%)$ were predicted to succumb to this malignancy (2). Novel therapies are required for the effective treatment of prostate cancer, and biomarkers based on the molecular biology of the disease are required for early diagnoses.

Aberrant expression or localization of $\beta$-catenin has been frequently observed in prostate tumor cells (3), thus suggesting that activation of the Wnt/ $\beta$-catenin signaling pathway may be involved in the development of prostate cancer. The Wnt/ $\beta$-catenin pathway regulates cell fate during embryonic development, and its activation is involved in the development of cancer (4-7). In the absence of Wnt signaling, cytoplasmic $\beta$-catenin levels are reduced due to destruction complex-induced degradation. The destruction complex consists of axin, adenomatous polyposis coli (APC), glycogen synthase kinase $3 \beta$ (GSK3 $\beta$ ) and casein kinase 1. Alterations that interfere with $\beta$-catenin degradation lead to the accumulation of $\beta$-catenin in the cytoplasm and subsequent translocation to the nucleus. In conjunction with the T-cell factor 
(TCF)/lymphoid enhancing factor transcription factors, $\beta$-catenin then induces cellular responses through the transcriptional activation of target genes.

Despite previous reports demonstrating that the $\mathrm{Wnt} / \beta$-catenin pathway is activated in prostate cancer (3), mutations in $A P C, \beta$-catenin (CTNNB1) and axin 1 (AXIN1), are rarely detected (The Cancer Genome Atlas Network; http://cancergenome.nih.gov/). Therefore, additional mechanisms may be responsible for the upregulation of $\beta$-catenin in prostate cancer. Frequently rearranged in advanced T-cell lymphomas-1 (FRAT1) overexpression activates the Wnt/ $\beta$-catenin pathway by binding to GSK3 $\beta$. Expression of FRAT1 is elevated in several types of human cancer, including ovarian cancer (8), esophageal cancer (9), glioma (10-12), astrocytoma (13), lung $(14,15)$ and pancreatic cancer (16). A recent report demonstrated that $\mathrm{N}$-myc downstream-regulated gene 1 regulates $\beta$-catenin phosphorylation and nuclear translocation through FRAT1 in prostate cancer cells (17), which indicates a potential association between FRAT1 and aberrant Wnt/ $\beta$-catenin signaling in prostate cancer. Therefore, the aim of the present study was to investigate the role of FRAT1 in prostate cancer.

\section{Materials and methods}

Prostate tissue microarray. A prostate tissue microarray (reference no. HProA100PG01), consisting of three normal prostate tissue samples and 59 prostate adenocarcinoma tissue samples, was obtained from Shanghai Outdo Biotech Co., Ltd. (Shanghai, China).

Cell culture, plasmid construction and transfection. The PC-3 and DU-145 human prostate cancer cell lines, and the NIH3T3 mouse embryonic fibroblast cell line were obtained from the American Type Culture Collection (Manassas, VA, USA). Cells were cultured in Dulbecco's modified Eagle's medium (Gibco; Thermo Fisher Scientific, Inc., Waltham, MA, USA), supplemented with $10 \%$ fetal bovine serum (Gibco; Thermo Fisher Scientific, Inc.), penicillin $(50 \mathrm{U} / \mathrm{ml})$ and streptomycin $(50 \mu \mathrm{g} / \mathrm{ml})$. Cell lines were maintained in a humidified incubator at $37^{\circ} \mathrm{C}$ and $5 \% \mathrm{CO}_{2}$. Culture medium was refreshed every 3 days and cells were subcultured when confluent.

The FRAT 1 overexpression plasmid (HA-FRAT1/pCEFL), FRAT1 short hairpin (sh) RNA knockdown plasmid (pSilencer-FRAT1), and control shRNA plasmid (pSilencer-control) were generated as described previously (9). TCF reporter plasmids, TOPFLASH and FOPFLASH were obtained from Upstate Biotechnology, Inc. (Lake Placid, NY, USA), and the pRL-TK wild-type control vector was purchased from Promega Corporation (Madison, WI, USA).

Transfections were performed in 24-well or 6-well plates. DU-145 or PC-3 cells $\left(2 \times 10^{5}\right)$ were seeded into each well of a 6-well tissue culture plate and were incubated for $24 \mathrm{~h}$. When the cells were $70-80 \%$ confluent, the culture media was removed and cells were washed with pre-warmed sterile phosphate-buffered saline. Lipofectamine 2000 Transfection Reagent (Invitrogen; Thermo Fisher Scientific, Inc.) was used to transfect cells with the plasmids according to the manufacturer's protocol. Cells were collected $24 \mathrm{~h}$ post-transfection and western blot analysis and reporter assays were performed.
To establish stable HA-FRAT1/pCEFL or pCEFL empty vector control cell lines, post-transfection, the medium was supplemented with $400 \mu \mathrm{g} / \mathrm{ml} \mathrm{G} 418$ and resistant clones were pooled and maintained in culture. Exogenous hemagglutinin (HA)-tagged FRAT1 expression was confirmed by western blot analysis.

Western blot analysis. Total protein was extracted from cultured cells using urea buffer $(8 \mathrm{M}$ urea, $1 \mathrm{M}$ thiourea, 0.5\% CHAPS, $50 \mathrm{mM}$ dithiothreitol and $24 \mathrm{mM}$ spermine). The protein concentration was determined using the Bradford Protein Assay Dye Reagent (cat. no. 500-0006; Bio-Rad Laboratories Inc., Hercules, CA, USA). The samples (40 $\mu \mathrm{g}$ of protein) were then separated by $12 \%$ sodium dodecyl sulfate-polyacrylamide gel electrophoresis and were transferred to nitrocellulose membranes. The membranes were blocked with $5 \%$ non-fat milk in Tris-buffered saline-Tween 20 at room temperature for $1 \mathrm{~h}$, and then incubated with primary antibodies overnight at $4^{\circ} \mathrm{C}$. The membranes were subsequently incubated for $1 \mathrm{~h}$ at room temperature with horseradish peroxidase (HRP)-conjugated secondary antibodies. Signals were detected using an enhanced chemiluminescence detection system (GE Healthcare Life Sciences, Chalfont, UK) and evaluated using ImageJ software (version, 1.42q; National Institutes of Health, Bethesda, MD, USA) (18). The following primary antibodies were used: Anti-HA (cat. no. sc-805; dilution, 1:1,000; Santa Cruz Biotechnology, Inc., Dallas, TX, USA), anti-FRAT1 (cat. no. ab108405; dilution, 1:1,000; Abcam, Cambridge, UK), anti- $\beta$-catenin (cat. no. 610153; 1:2,000; BD Biosciences, Franklin Lakes, NJ, USA), anti-phosphorylated (p)- $\beta$-catenin (Ser33/37/Thr41; cat. no. 9561; dilution, 1:1,000; Cell Signaling Technology, Inc., Danvers MA, USA), anti- $\beta$-actin (cat. no. A3854; dilution, 1:5,000; Sigma-Aldrich; Merck Millipore, Darmstadt, Germany) and anti- $\beta$-tubulin (cat. no. ab6046; dilution, 1:5,000, Abcam, MA, USA). The following HRP-conjugated secondary antibodies were used: Goat anti-rabbit immunoglobulin G (IgG) H\&L (cat. no. ab97051; dilution, 1:1,000; Abcam), goat anti-mouse IgG H\&L (cat. no. ab6789; dilution, 1:1,000; Abcam).

Cell growth assay. Cells that were stably or transiently transfected were harvested and re-plated in triplicate in 24-well plates. Cells were counted every 2 days with a hemocytometer, and a Trypan blue exclusion assay was used to assess cell viability. In brief, $0.1 \mathrm{ml}$ Trypan blue stock solution was mixed with $0.1 \mathrm{ml}$ cell suspension before loading onto a hemocytometer. Cells were counted immediately under a light microscope at low magnification.

Immunohistochemical analysis. The prostate tissue microarray was deparaffinized, rehydrated and incubated with $3 \%$ hydrogen peroxide for $10 \mathrm{~min}$ to block endogenous peroxidase activity. The slide was subsequently blocked with normal goat serum at room temperature for $1 \mathrm{~h}$, and then incubated at $4^{\circ} \mathrm{C}$ overnight with a primary antibody against FRAT1 (cat. no. ab137391; dilution, 1:400; Abcam, Cambridge, UK). The slide was then incubated with a biotinylated goat anti-rabbit IgG H\&L secondary antibody (dilution, 1:250; cat. no. BP-9100, Vector Laboratories, Inc., Burlingame, CA, USA) for $40 \mathrm{~min}$ at room temperature, and subsequently stained with 
the VECTASTAIN Elite ABC HRP kit (Vector Laboratories, Inc.) followed by counterstaining with hematoxylin. The localization of FRAT1 in each sample spot within the tissue microarray slide was categorized as membranous, cytoplasmic or nuclear. The staining intensity was graded as follows: No staining (0), weak (1), moderate (2) and intense staining (3). Samples that could not be graded were scored as 'not applicable'. For each sample, FRAT1 expression was determined as the average score of the sample spot, and then further subgrouped into low (score 0) or high (scores 1-3) FRAT1 expression.

Reporter assays. Following transfection with either TOPFLASH (100 ng) or FOPFLASH (100 ng), the internal control plasmid pRL-TK (5 ng) and HA-FRAT1/pCEFL, pCEFL, pSilencer-FRAT1 or pSilencer-control-transfected cells were cultured for $36 \mathrm{~h}$ at $37^{\circ} \mathrm{C}$ before they were lysed using Passive Lysis Buffer (cat. no. E1941; Promega Corporation) to measure the luciferase reporter activity using the Dual-Luciferase Reporter Assay system (Promega Corporation) according to the manufacturer's instructions. Firefly luciferase activity was normalized to Renilla luciferase activity. Data are presented as the mean \pm standard deviation (SD) for independent triplicate cultures.

Tumor growth in nude mice. NIH3T3/control or NIH3T3/FRAT1 $\left(4 \times 10^{6}\right)$ cells were prepared in $0.2 \mathrm{ml}$ saline, and were injected bilaterally and subcutaneously, each into the left and right forelegs of four female nude mice (age, 4-6 weeks; weight, 16-20 g; Beijing HFK Bioscience Co., Ltd.). Prior to tumor cell implantation, mice were allowed to acclimatize to laboratory conditions for 3 days. The mice were housed in a pathogen-free environment and monitored every 2 days. Animals had free access to standard food and water, and were maintained in $12 \mathrm{~h} \mathrm{light/dark} \mathrm{cycles} \mathrm{throughout} \mathrm{the} \mathrm{course} \mathrm{of}$ treatment. At the end of the experiment, the mice were sacrificed by cervical dislocation. The date when a palpable tumor first arose and the weight of the removed tumor were recorded. The mice were treated in accordance with the Regulations of Laboratory Animal Quality issued by the Chinese Ministry of Science and Technology (Beijing, China). Animal experiments were approved by the Institutional Animal Care and Use Committee of Cancer Hospital, Chinese Academy of Medical Sciences (reference no. NCC2015A019).

Statistical analysis. Data are presented as the mean \pm SD. A two-tailed, unpaired Student's t-test was used to compare independent samples from two groups. Data were analyzed using the SPSS software program (version 16.0; SPSS, Inc., Chicago, IL, USA). $\mathrm{P}<0.05$ was considered to indicate a statistically significant difference.

\section{Results}

FRAT1 is expressed exclusively in the nuclei of normal prostate basal cells and is overexpressed in human prostate cancer. FRATl mRNA expression in established human cell lines was first investigated using the Human Protein Atlas database (http://www.proteinatlas. org/ENSG00000165879-FRAT1/cell). Notably, FRAT1

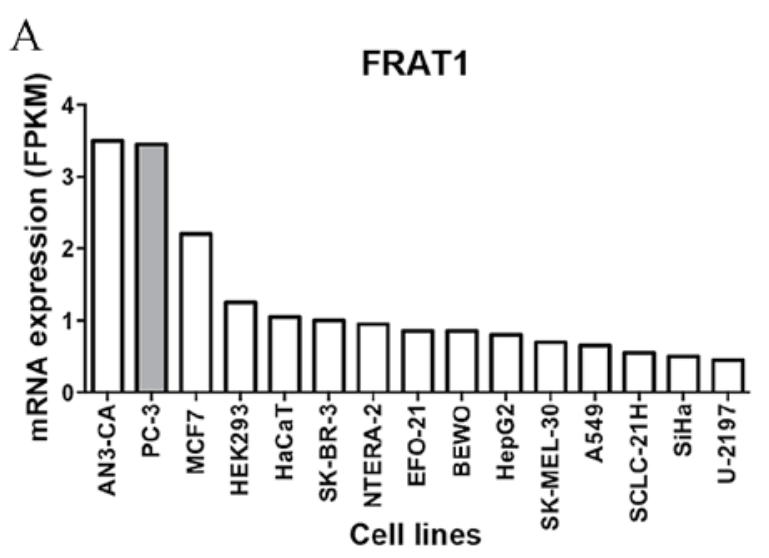

B

FRAT1

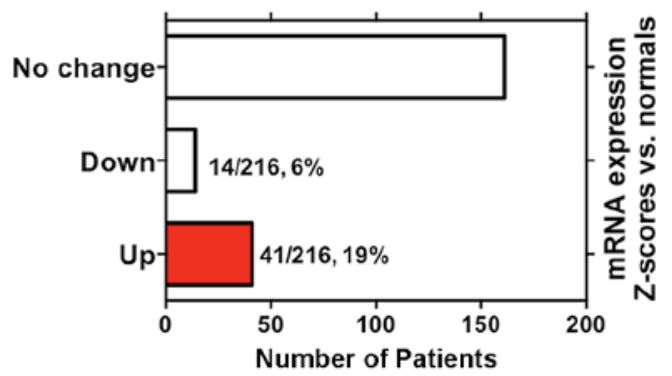

Figure 1. FRAT1 mRNA expression in established human cell lines and patients with prostate cancer. (A) FRAT1 mRNA expression in established human cancer cell lines as determined using the human protein atlas protein database (http://www.proteinatlas.org/ENSG00000165879-FRAT1/cell). (B) FRAT1 mRNA expression mRNA expression Z-Scores vs. normal threshold \pm 1.0 in patients with prostate cancer as determined using. The cancer genome atlas cBioPortal database (Memorial Sloan Kettering Cancer Center; http://www.cbioportal.org/). FRAT1, frequently rearranged in advanced T-cell lymphomas-1; FPKM, fragments/kilobase of transcript/million mapped reads.

mRNA expression levels in PC-3 prostate cancer cells were observed to be among the highest across all of the cell lines included in the analysis (Fig. 1A).

In order to explore the clinical implications of FRAT1 expression in prostate cancer, data from The Cancer Genome Atlas cBioPortal database (http://www.cbioportal.org/) were analyzed (19-21). As shown in Fig. 1B, upregulation of FRATI mRNA expression levels was frequent in patients with prostate adenocarcinoma (41/216, 19\%; Memorial Sloan Kettering Cancer Center; http://www.cbioportal.org/study?id=prad_ mskcc\#summary).

The protein expression of FRAT1 in normal human prostate tissue and prostate adenocarcinoma tissues was analyzed by immunohistochemical analysis using a human prostate cancer tissue microarray. Expression of FRAT1 was observed in all three cases of normal prostate epithelium, exclusively in the nuclei of basal cells (Fig. 2A). These results are consistent with the in situ hybridization results of a previous study, demonstrating that FRAT1 protein expression was present in all samples of normal esophageal squamous cell epithelium and in the basal layers (9). In the present study, nuclear FRAT1 expression was detected in $68 \%(40 / 59)$ of prostate adenocarcinoma samples (Fig. 2B). Since only a small fraction of cells (basal cells) in the normal 

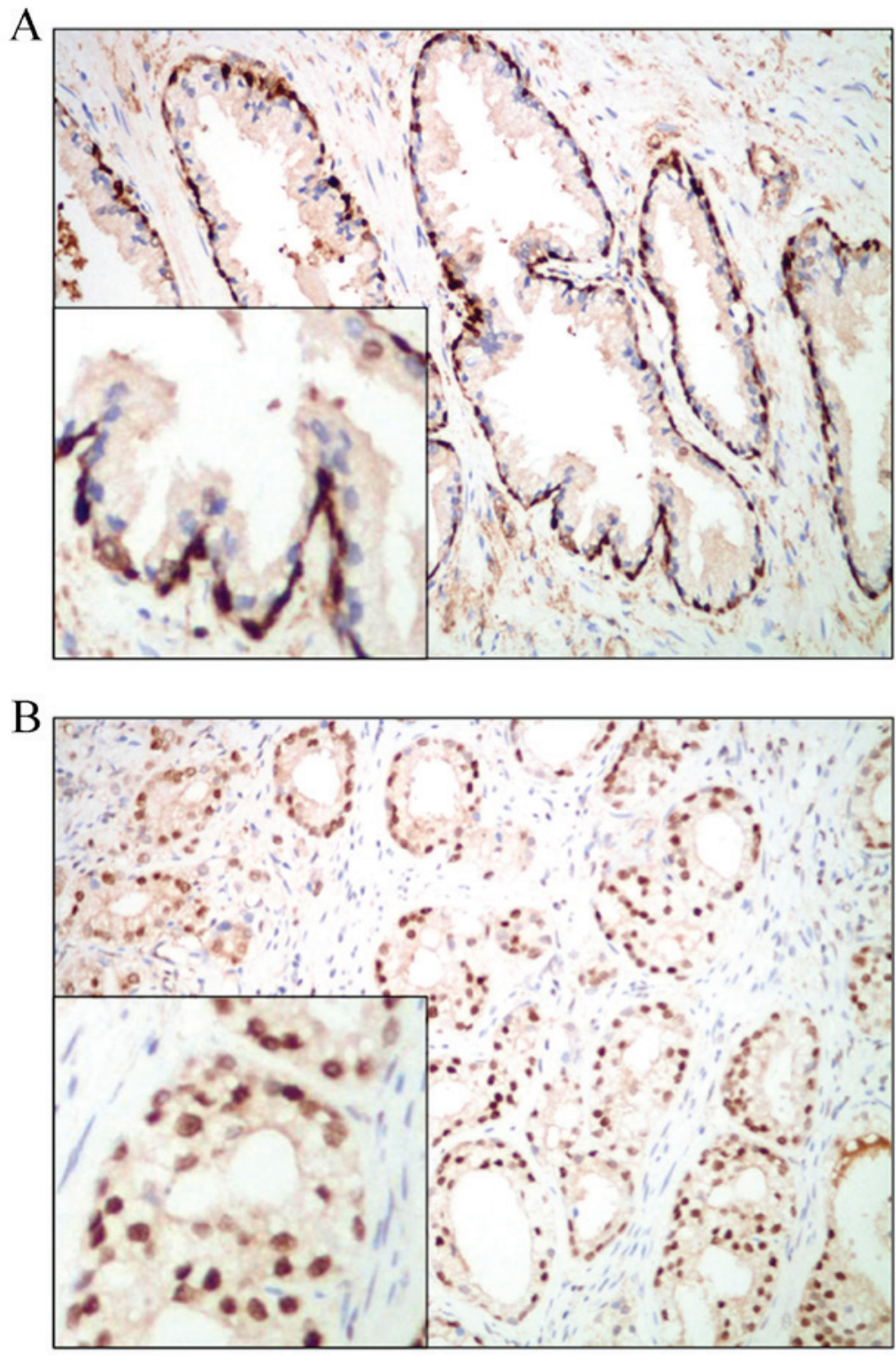

Figure 2. FRAT1 is expressed exclusively in the nuclei of normal prostate basal cells and is overexpressed in human prostate cancer. Representative FRAT1 expression patterns in (A) normal prostate and (B) prostate adenocarcinoma tissues from a tissue microarray following incubation with an anti-FRAT1 antibody and staining with 3,3-diaminobenzidine (magnification, x200; inset magnification, x400). FRAT1, frequently rearranged in advanced T-cell lymphomas-1.

prostate tissue samples were observed to express FRAT1, this protein was determined to be overexpressed in prostate adenocarcinoma tissues.

FRAT1 expression status affects prostate cancer cell growth. The next aim of the present study was to investigate whether the expression status of FRAT1 influences the growth of human prostate cancer cell lines. As determined using the Catalogue of Somatic Mutations in Cancer database (http:/cancer.sanger.ac.uk/cosmic), FRAT1 mRNA expression levels in PC-3 cells were markedly higher when compared with DU-145 cells (Fig. 3A).

Forced overexpression of FRAT1 markedly promoted the growth rate of DU-145 cells, with a 4-fold increase observed at day $6(\mathrm{P}<0.001$; Fig. 3B). Conversely, knockdown of FRAT1 expression by RNA interference (RNAi) significantly inhibited the cell growth rate of PC-3 cells, with a $30-50 \%$ decrease at days $4-6(\mathrm{P}=0.003$ at day $4 ; \mathrm{P}<0.001$ at days 5 and 6; Fig. 3C). These data indicate that increased FRAT1 expression may provide a growth advantage in prostate cancer cells.

FRATl activates $\beta$-catenin-dependent transcriptional activity. It has previously been reported that FRAT1 positively regulates the activity of the $\mathrm{Wnt} / \beta$-catenin signaling pathway (17). In order to investigate whether this process may also occur in prostate cancer cells, the effect of FRAT1 expression on the transcriptional activity of $\beta$-catenin was investigated using a TOPFLASH/FOPFLASH reporter assay. This assay consists of wild type (TOP) or mutated (FOP) binding sites for the $\beta$-catenin/TCF complex upstream of a minimal thymidine kinase promoter and luciferase open reading frame.

Overexpression of FRAT1 in DU-145 cells significantly increased TOPFLASH activity, with a 3-fold increase compared to the control cells $(\mathrm{P}<0.001$; Fig. 4A). A minimal effect of FRAT1 overexpression on the FOPFLASH reporter 
A

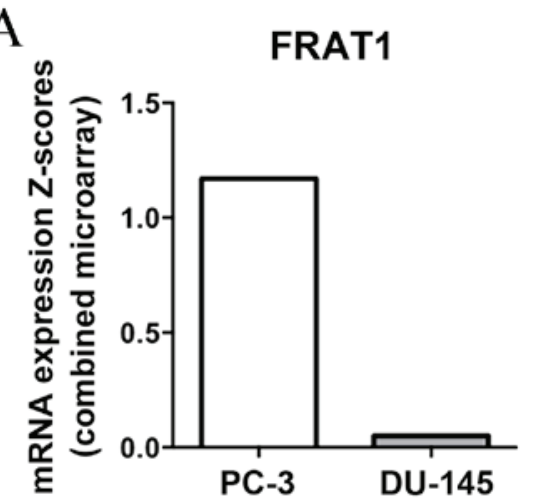

$\mathrm{B}$

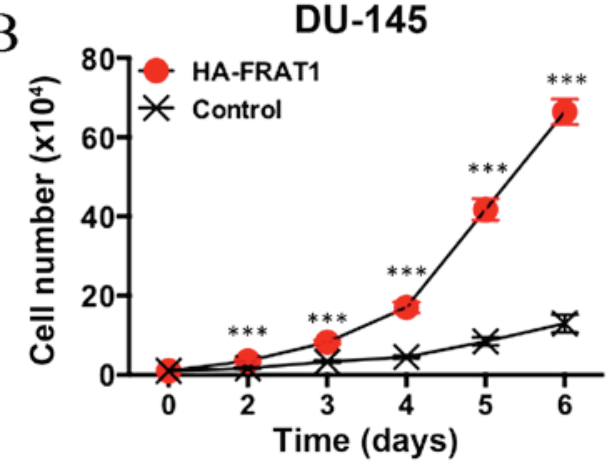

C

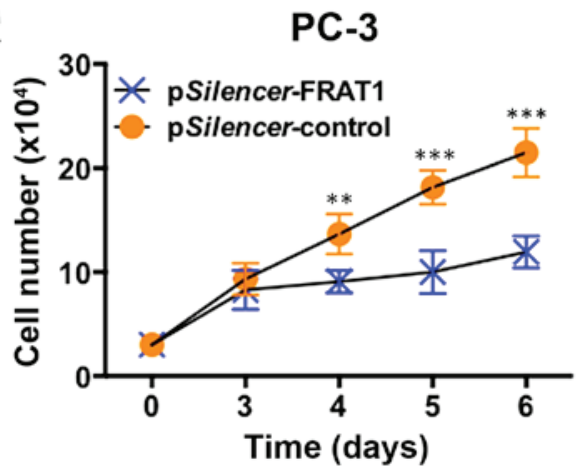

Figure 3. Effects of FRAT1 expression on prostate cancer cell growth. (A) FRAT1 mRNA expression in PC-3 and DU-145 cells as determined using the catalogue of somatic mutations in cancer database (http://cancer.sanger.ac.uk/cosmic). Cell growth rates of (B) DU-145 cells following transfection with HA-FRAT1 or control expression vectors, and (C) PC-3 cells following transfection with pSilencer-FRAT1 or pSilencer-control shRNA. Transfected cells were collected and replated at a density of 50 cells $/ \mathrm{mm}^{2}$ in triplicate. Cell number was assessed each day for 6 days. Trypan blue exclusion assay was used to assess cell viability. Data are presented as the mean \pm standard deviation. HA-FRAT1, hemagglutinin-tagged frequently rearranged in advanced T-cell lymphomas-1; shRNA, short hairpin RNA. ${ }^{* *} \mathrm{P}<0.01$ and ${ }^{* * * *} \mathrm{P}<0.001$ vs. control.

was detected (data not shown). In addition, knockdown of FRAT1 in PC-3 cells using RNAi, markedly inhibited $\beta$-catenin/TCF-dependent transcriptional activity, with a $>70 \%$ reduction in TOPFLASH activity $(\mathrm{P}<0.001 ;$ Fig. $4 \mathrm{~B})$.

Overexpression of FRAT1 in DU-145 cells inhibited the expression of $\beta$-catenin phosphorylated at Ser33/37 and Thr41 residues, which provides an explanation for the observed increase in total $\beta$-catenin levels (Fig. 4A). Conversely, depletion of FRAT1 in PC-3 cells resulted in an increase in the protein expression levels of $\mathrm{p}-\beta$-catenin (at Ser33/37 and Thr41

residues), and decreased levels of total $\beta$-catenin expression (Fig. 4B). These data confirm that FRAT1 may function to stabilize $\beta$-catenin in prostate cancer cells.

FRAT1 overexpression in NIH3T3 cells induces tumor formation in nude mice. The in vitro results indicated that FRAT1 may affect prostate cancer cell growth. In order to confirm the tumorigenic potential of FRAT1 in vivo, tumor xenograft experiments involving four nude mice were performed using NIH3T3 cells stably transfected with FRAT1 (NIH3T3/FRAT1) or control vectors (NIH3T3/control; Fig. 5A). At 8 weeks following injection with the transfected cells, the mice were sacrificed and the tumors were removed and weighed. Tumors developed in all four nude mice at the right lateral site where the NIH3T3/FRAT1 cells were injected (Fig. 5B and Table I). Conversely, no palpable tumors developed on the left lateral side where NIH3T3/control cells were injected (Fig. 5B and Table I). At 3 weeks following injection with NIH3T3/FRAT1, palpable nodular neoplasms developed and tumors were observed at week 5 . These data provide evidence to suggest that FRAT1 serves a tumorigenic role in vivo, as its overexpression in normal NIH3T3 mouse fibroblast cells led to the formation of tumors in nude mice.

\section{Discussion}

Prostate cancer is the fifth most common cause of cancer-associated mortality worldwide in men, and is the eighth most common cause of cancer-associated mortality overall (1). Molecular biomarkers and personalized treatment strategies based on an improved understanding of the molecular mechanisms underlying prostate cancer development and progression would maximize therapeutic outcome (22). Despite previous reports demonstrating that a large proportion of prostate tumor cells exhibit aberrant expression or localization of $\beta$-catenin (3), the observation that $C T N N B 1, A P C$ and AXINI are rarely mutated in prostate cancer suggests that genetic alterations may not be responsible for activation of the Wnt/ק-catenin pathway (3). The results of the present study demonstrated that FRAT1 may contribute to the activation of the $\beta$-catenin/TCF pathway in prostate cancer cells, due to the observation that FRAT1 activated the $\beta$-catenin/TCF promoter luciferase reporter gene in DU-145 prostate cancer cells. In addition, FRAT1 was observed to affect the growth of prostate cancer cells in vitro. Furthermore, forced expression of FRAT1 in NIH3T3 cells was sufficient to induce cell transformation and lead to tumor growth in vivo. These findings suggested that FRAT1 exhibits oncogenic properties in prostate cancer.

FRAT1 is overexpressed in prostate cancer. In normal prostate tissue, FRAT1 is expressed exclusively in the nuclei of basal cells. At a histological level, the human prostate primarily consists of epithelial and stromal cells. In the epithelial cell layer, there are four differentiated cell types, including basal, secretory luminal, neuroendocrine and transit-amplifying cells. These cells display distinct morphologies, locations, functions and expression markers. The basal cells, where adult prostate stem cells are thought to reside, form a layer of flattened cuboidal-shaped cells above the basement membrane (23). In 2014, Goksel et al (24) reported 
Table I. FRAT1 overexpression in NIH3T3 cells induces tumor formation in nude mice.

\begin{tabular}{|c|c|c|c|c|c|c|}
\hline \multirow[b]{2}{*}{$\begin{array}{l}\text { No. of } \\
\text { cells }\end{array}$} & \multicolumn{3}{|c|}{ NIH3T3/FRAT1 } & \multicolumn{3}{|c|}{ NIH3T3/control } \\
\hline & $\begin{array}{c}\text { Tumor weight } \\
\text { (mg) }\end{array}$ & $\begin{array}{l}\text { No. tumors/ } \\
\text { injection }\end{array}$ & $\begin{array}{l}\text { Latency }^{\mathrm{b}} \\
\text { (days) }\end{array}$ & $\begin{array}{l}\text { Tumor weight } \\
\text { (mg) }\end{array}$ & $\begin{array}{l}\text { No. tumors/ } \\
\text { injection }\end{array}$ & $\begin{array}{c}\text { Latency }^{b} \\
\text { (days) }\end{array}$ \\
\hline $4 \times 10^{6}$ & $2105 \pm 409$ & $4 / 4$ & $21-35$ & 0 & $0 / 4$ & $>56$ \\
\hline
\end{tabular}

Tumor weight is expressed as the mean \pm standard deviation. ${ }^{\mathrm{N}}$ Number of cells that were injected subcutaneously and bilaterally into four nude mice. ${ }^{b}$ Latency is defined as the time interval required for palpable tumors to arise. FRAT1, frequently rearranged in advanced T-cell lymphomas-1.
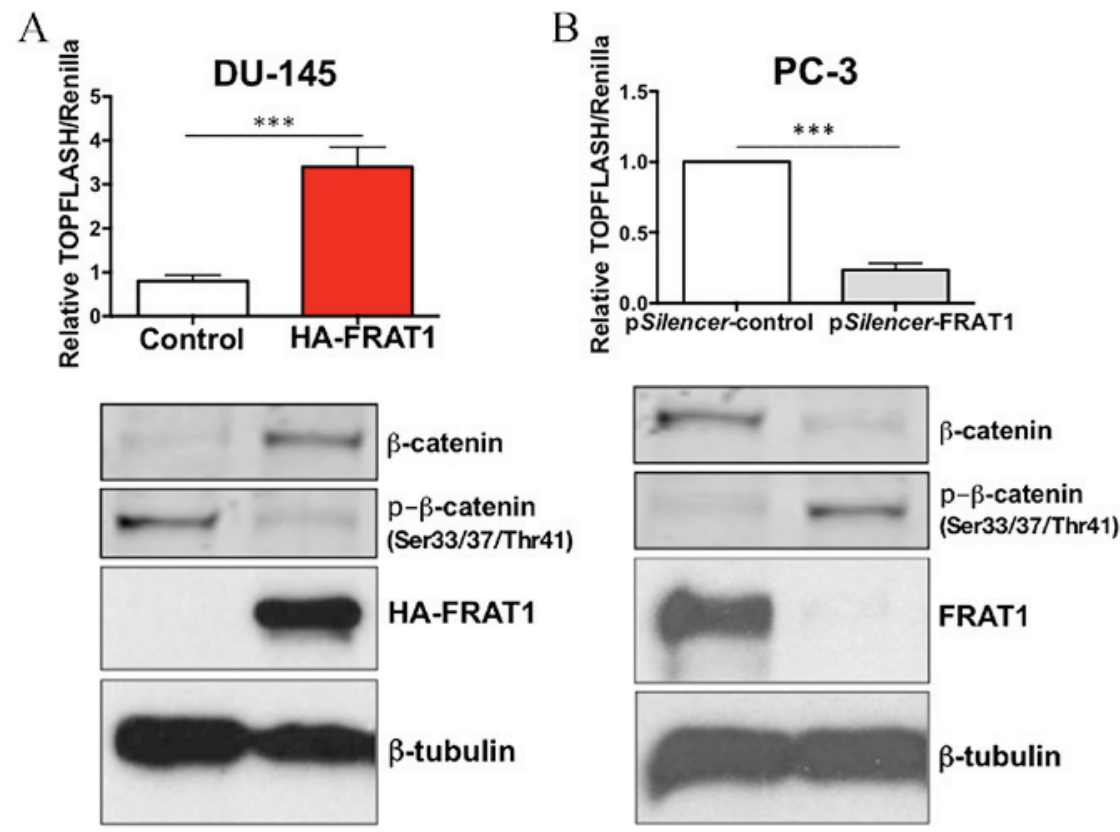

Figure 4. Effects of FRAT1 on $\beta$-catenin/TCF activity. TOPFLASH $\beta$-catenin/TCF complex binding site reporter assay analysis and western blot analysis of $\beta$-catenin, p- $\beta$-catenin (Ser33/37/Thr41) and HA-FRAT1/FRAT1 expression in (A) DU-145 cells following transfection with HA-FRAT1 or control expression vectors, and (B) PC-3 cells following transfection with pSilencer-FRAT1 or pSilencer-control shRNA. $\beta$-tubulin was used as a loading control for western blot analysis. To normalize transfection efficiency, pRL-TK Renilla luciferase reporter constructs were cotransfected in each sample. Luciferase reporter activity in cells transfected with control vectors was set as 1.0. Experiments were performed in triplicate and data are presented as the mean \pm standard deviation. TCF, T-cell factor; $\mathrm{p}$ - $\beta$-catenin, phosphorylated $\beta$-catenin; HA-FRAT1, hemagglutinin-tagged frequently rearranged in advanced T-cell lymphomas-1; shRNA, short hairpin RNA. ${ }^{* * *} \mathrm{P}<0.001$ vs. control.

A

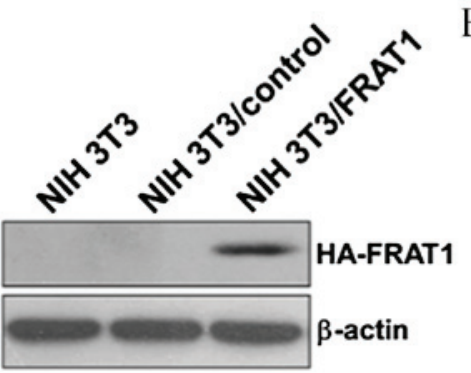

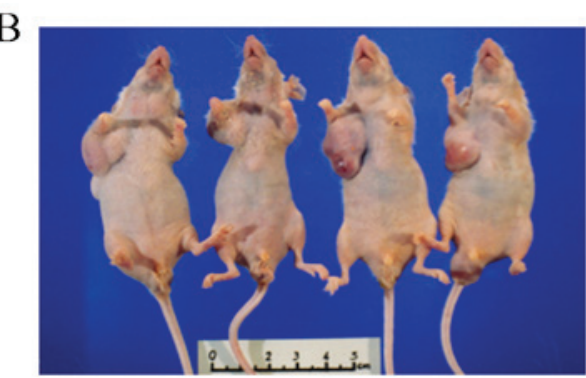

Figure 5. FRAT1 overexpression in NIH3T3 cells induces tumor formation in nude mice. (A) Western blot analysis of HA-FRAT1 expression in NIH3T3 parental, NIH3T3/control or NIH3T3/FRAT1 cells. $\beta$-actin was used as a loading control. (B) An image showing tumor growth in nude mice at 8 weeks following injection with NIH3T3/FRAT1 (right lateral) or NIH3T3/control cells (left lateral). HA-FRAT1, hemagglutinin-tagged frequently rearranged in advanced T-cell lymphomas-1.

that Wnt pathway-associated genes, including FRAT1, were significantly upregulated in the CD133 ${ }^{\text {high }} / \mathrm{CD} 44^{\text {high }}$ cancer stem cell (CSC) monolayer group when compared with non-CSC counterparts. Future studies will be required to 
confirm whether FRAT1 is a potential marker for the stemness of prostate basal cells.

The human FRATl gene is a homologue of the mouse proto-oncogene Fratl, which was demonstrated to convey a selective advantage to cells at the later stages of murine T-cell lymphomagenesis $(25,26)$. FRAT1 functions as a GSK3-binding protein, which is similar to the function of its Xenopus homolog GSK3-binding protein (27). Ectopic expression of Frat1 in Xenopus embryos induces secondary axis formation by stabilizing $\beta$-catenin levels (28). Overexpression of Fratl in mouse cells induces $\beta$-catenin/TCF-dependent reporter gene activity (29). In addition, Fratl interacts with Dishevelled (Dvl), which may enable signaling from Dvl to GSK3 (30). Finally, low-density lipoprotein receptor related protein 5 recruits axin and Frat1 to the cell membrane, which induces axin degradation and Frat1-mediated inhibition of GSK-3. As a consequence, $\beta$-catenin is not targeted for degradation, which leads to the activation of the Wnt/ $\beta$-catenin signaling pathway (31-33).

In the present study, the nuclear expression patterns of FRAT1 in normal prostate basal cells and prostate tumor cells were reported. Although GSK3 $\beta$ generally functions as part of the $\beta$-catenin-degradation complex in the cytosol, there is evidence to suggest that GSK $3 \beta$ may also reduce Wnt signaling in the nucleus (34). Caspi et al (34) demonstrated that GSK3 $\beta$ is able to translocate to the nucleus, where it forms a complex with $\beta$-catenin and reduces the level of $\beta$-catenin/TCF-dependent transcriptional activity. The results of the present study suggest that nuclear FRAT1 activates the Wnt/ $\beta$-catenin signaling pathway and confers an increase in prostate cancer cell growth, potentially by preventing nuclear GSK $3 \beta$-mediated inhibition of $\beta$-catenin/TCF activity.

\section{Acknowledgements}

The present study was supported by grants from the National Natural Science Youth Foundation (grant nos. 81201779 and 81502118), the Natural Science Foundation of Hubei Province (grant no. 2014CFB250) and the National Natural Science Foundation of China (grant nos. 81452761 and 81321091). Dr Yihua Wang was supported by Biological Sciences, Faculty of Natural and Environmental Sciences, University of Southampton.

\section{References}

1. Torre LA, Bray F, Siegel RL, Ferlay J, Lortet-Tieulent J and Jemal A: Global cancer statistics, 2012. CA Cancer J Clin 65: 87-108, 2015.

2. Siegel RL, Miller KD and Jemal A: Cancer statistics, 2015. CA Cancer J Clin 65: 5-29, 2015.

3. Kypta RM and Waxman J: Wnt/beta-catenin signalling in prostate cancer. Nat Rev Urol 9: 418-428, 2012.

4. Clevers H: Wnt/beta-catenin signaling in development and disease. Cell 127: 469-480, 2006.

5. Reya T and Clevers H: Wnt signalling in stem cells and cancer. Nature 434: 843-850, 2005.

6. Barker $\mathrm{N}$ and Clevers $\mathrm{H}$ : Mining the Wnt pathway for cancer therapeutics. Nat Rev Drug Discov 5: 997-1014, 2006.

7. Kahn M: Can we safely target the WNT pathway? Nat Rev Drug Discov 13: 513-532, 2014.

8. Wang Y, Hewitt SM, Liu S, Zhou X, Zhu H, Zhou C, Zhang G, Quan L, Bai J and Xu N: Tissue microarray analysis of human FRAT1 expression and its correlation with the subcellular localisation of beta-catenin in ovarian tumours. Br J Cancer 94: 686-691, 2006.
9. Wang Y, Liu S, Zhu H, Zhang W, Zhang G, Zhou X, Zhou C, Quan L, Bai J, Xue L, et al: FRAT1 overexpression leads to aberrant activation of beta-catenin/TCF pathway in esophageal squamous cell carcinoma. Int J Cancer 123: 561-568, 2008.

10. Guo G, Mao X, Wang P, Liu B, Zhang X, Jiang X, Zhong C, Huo J, Jin J and Zhuo Y: The expression profile of FRAT1 in human gliomas. Brain Res 1320: 152-158, 2010.

11. Guo G, Kuai D, Cai S, Xue N, Liu Y, Hao J, Fan Y, Jin J, Mao X, Liu B, et al: Knockdown of FRAT1 expression by RNA interference inhibits human glioblastoma cell growth, migration and invasion. PloS One 8: e61206, 2013.

12. Guo G, Zhong CL, Liu Y, Xue N, Liu Y, Hao J, Fan Y, Jin J, Mao X and Liu B: Overexpression of FRAT1 is associated with malignant phenotype and poor prognosis in human gliomas. Dis Markers 2015: 289750, 2015.

13. Guo G, Liu B, Zhong C, Zhang X, Mao X, Wang P, Jiang X, Huo J, Jin J, Liu X and Chen X: FRAT1 expression and its correlation with pathologic grade, proliferation, and apoptosis in human astrocytomas. Med Oncol 28: 1-6, 2011.

14. Zhang Y, Yu JH, Lin XY, Miao Y, Han Y, Fan CF, Dong XJ, Dai SD and Wang EH: Overexpression of Frat1 correlates with malignant phenotype and advanced stage in human non-small cell lung cancer. Virchows Arch 459: 255-263, 2011.

15. Zhang Y, Han Y, Zheng R, Yu JH, Miao Y, Wang L and Wang EH: Expression of Frat 1 correlates with expression of $\beta$-catenin and is associated with a poor clinical outcome in human SCC and AC. Tumour Biol 33: 1437-1444, 2012.

16. Yuan Y, Yang Z, Miao X, Li D, Liu Z and Zou Q: The clinical significance of FRAT1 and ABCG2 expression in pancreatic ductal adenocarcinoma. Tumour Biol 36: 9961-9968, 2015.

17. Jin R, Liu W, Menezes S, Yue F, Zheng M, Kovacevic Z and Richardson DR: The metastasis suppressor NDRG1 modulates the phosphorylation and nuclear translocation of $\beta$-catenin through mechanisms involving FRAT1 and PAK4. J Cell Sci 127: 3116-3130, 2014.

18. Schneider CA, Rasband WS and Eliceiri KW: NIH Image to ImageJ: 25 years of image analysis. Nat Methods 9: 671-675, 2012.

19. Taylor BS, Schultz N, Hieronymus H, Gopalan A, Xiao Y, Carver BS, Arora VK, Kaushik P, Cerami E, Reva B, et al: Integrative genomic profiling of human prostate cancer. Cancer Cell 18: 11-22, 2010.

20. Gao J, Aksoy BA, Dogrusoz U, Dresdner G, Gross B, Sumer SO, Sun Y, Jacobsen A, Sinha R, Larsson E, et al: Integrative analysis of complex cancer genomics and clinical profiles using the cBioPortal. Sci Signal 6: pl1, 2013.

21. Cerami E, Gao J, Dogrusoz U, Gross BE, Sumer SO, Aksoy BA, Jacobsen A, Byrne CJ, Heuer ML, Larsson E, et al: The cBio cancer genomics portal: An open platform for exploring multidimensional cancer genomics data. Cancer Discov 2: 401-404, 2012.

22. Chesire DR and Isaacs WB: Beta-catenin signaling in prostate cancer: An early perspective. Endocr Relat Cancer 10: 537-560, 2003.

23. Prajapati A, Gupta S, Mistry B and Gupta S: Prostate stem cells in the development of benign prostate hyperplasia and prostate cancer: Emerging role and concepts. Biomed Res Int 2013: 107954, 2013.

24. Goksel G, Bilir A, Uslu R, Akbulut H, Guven U and Oktem G: WNT1 gene expression alters in heterogeneous population of prostate cancer cells; decreased expression pattern observed in CD133+/CD44+ prostate cancer stem cell spheroids. J BUON 19: 207-214, 2014.

25. Jonkers J, Korswagen HC, Acton D, Breuer M and Berns A: Activation of a novel proto-oncogene, Frat 1, contributes to progression of mouse T-cell lymphomas. EMBO J 16: 441-450, 1997.

26. Jonkers J, Weening JJ, van der Valk M, Bobeldijk R and Berns A: Overexpression of Frat1 in transgenic mice leads to glomerulosclerosis and nephrotic syndrome, and provides direct evidence for the involvement of Fratl in lymphoma progression. Oncogene 18: 5982-5990, 1999.

27. Yost C, Farr GH III, Pierce SB, Ferkey DM, Chen MM and Kimelman D: GBP, an inhibitor of GSK-3, is implicated in Xenopus development and oncogenesis. Cell 93: 1031-1041, 1998.

28. Jonkers J, van Amerongen R, van der Valk M, Robanus-Maandag E, Molenaar M, Destrée O and Berns A: In vivo analysis of Frat1 deficiency suggests compensatory activity of Frat3. Mech Dev 88: 183-194, 1999.

29. van Amerongen R, van der Gulden H, Bleeker F, Jonkers J and Berns A: Characterization and functional analysis of the murine Frat2 gene. J Biol Chem 279: 26967-26974, 2004. 
30. Li L, Yuan H, Weaver CD, Mao J, Farr GH III, Sussman DJ, Jonkers J, Kimelman D and Wu D: Axin and Frat1 interact with dvl and GSK, bridging Dvl to GSK in Wnt-mediated regulation of LEF-1. EMBO J 18: 4233-4240, 1999.

31. Hay E, Faucheu C, Suc-Royer I, Touitou R, Stiot V, Vayssière B, Baron R, Roman-Roman S and Rawadi G: Interaction between LRP5 and Fratl mediates the activation of the Wnt canonical pathway. J Biol Chem 280: 13616-13623, 2005.

32. Thomas GM, Frame S, Goedert M, Nathke I, Polakis P and Cohen P: A GSK3-binding peptide from FRAT1 selectively inhibits the GSK3-catalysed phosphorylation of axin and beta-catenin. FEBS Lett 458: 247-251, 1999.
33. Hagen T, Cross DA, Culbert AA, West A, Frame S, Morrice N and Reith AD: FRAT1, a substrate-specific regulator of glycogen synthase kinase-3 activity, is a cellular substrate of protein kinase A. J Biol Chem 281: 35021-35029, 2006.

34. Caspi M, Zilberberg A, Eldar-Finkelman $H$ and Rosin-Arbesfeld R: Nuclear GSK-3beta inhibits the canonical Wnt signalling pathway in a beta-catenin phosphorylation-independent manner. Oncogene 27: 3546-3555, 2008. 